07

\title{
Analysis of Fringe Field Formed Inside LDA Measurement Volume Using Compact Two Hololens Imaging Systems*
}

\author{
(C) Abhijit Ghosh ${ }^{1}$, A.K. Nirala ${ }^{1}$, H.L. Yadav ${ }^{2}$ \\ ${ }^{1}$ Biomedical Optics Lab, Department of Applied Physics, \\ Indian Institute of Technology (Indian School of Mines), Dhanbad \\ 826004 Jharkhand, India \\ ${ }^{2}$ Photonics Lab, Department of Physics, National Institute of Technology, Jamshedpur \\ 831014 Jharkhand, India \\ e-mail: abhi.photonics@gmail.com, aknirala@gmail.com, hly_physics@rediffmail.com
}

Received April 03, 2017; in final form, October 24, 2017

We have designed and fabricated four LDA optical setups consisting of aberration compensated four different compact two hololens imaging systems. We have experimentally investigated are realized a hololens recording geometry which is interferogram of converging spherical wavefront with mutually coherent planar wavefront. Proposed real time monitoring and actual fringe field analysis techniques allow complete characterizations of fringes formed at measurement volume and permit to evaluate beam quality, alignment and fringe uniformity with greater precision. After experimentally analyzing the fringes formed at measurement volume by all four imaging systems, it is found that fringes obtained using compact two hololens imaging systems get improved both qualitatively and quantitatively compared to that obtained using conventional imaging system. Results indicate qualitative improvement of non uniformity in fringe thickness and micro intensity variations perpendicular to the fringes, and quantitative improvement of $39.25 \%$ in overall average normalized standard deviations of fringe width formed by compact two hololens imaging systems compare to that of conventional imaging system.

DOI: $10.21883 /$ OS.2018.03.45665.84-17

* Полный текст статьи опубликован в английской версии журнала. 